\title{
Myocardial Injury during COVID 19: Pembrolizumab Induced Myocarditis or Coronavirus NL63 Myocardial Damage?
}

\author{
De Paolis $\mathbf{M}^{1}$, Camilli $\mathrm{G}^{1}$, Mezzetti $\mathrm{P}^{1}$, Bier $\mathbf{N}^{1}$, Faleburle $\mathrm{A}^{1}$, Di Giuli $\mathrm{C}^{2}$, \\ Saraca $\mathrm{LM}^{2 *}$, Sicari $\mathrm{F}^{3}$, Palumbo $\mathbf{M}^{2}$ and Dominici $\mathbf{M}^{1}$ \\ ${ }^{1}$ Complex Structure of Cardiology, Italy \\ ${ }^{2}$ Clinic of Infectious Diseases, Italy \\ ${ }^{3}$ Department of Medicine, University of Perugia, Italy
}

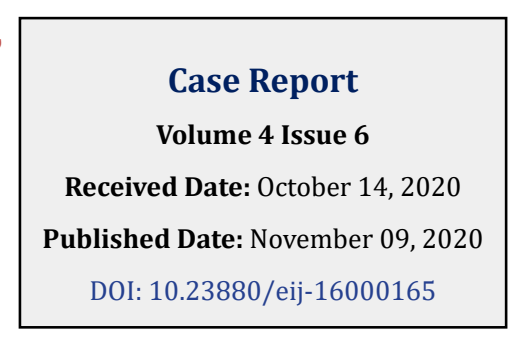

*Corresponding author: Lavinia Maria Saraca, Clinic of Infectious Diseases, A.O. S. Maria, Tristano Di Joannuccio, Terni, Italy, Tel: 3290713368; Email: lm.saraca@aospterni.it

\section{Abstract}

Myocarditis is an inflammatory pathology of the heart muscle having heterogeneous etiology: infectious, autoimmune (e.g. Rheumatoid Arthritis, Sarcoidosis, Systemic Lupus Erythematosus, and Scleroderma) or iatrogenic for exposure to toxins or drugs (e.g. cardiotoxicity from chemotherapy). Myocarditis presents itself with a wide spectrum of clinical manifestations, ranging from the infarct-like forms, heart failure, syncope up to hypo or hyperkinetic arrhythmias, or run in a paucisymptomatic way with almost silent symptoms. The most common cause of myocarditis is a viral infection. The coronavirus SARS-CoV2, the causative agent of COVID 19, which is responsible for the current pandemic, has a close correlation with myocardial damage and the increased risk of cardiovascular events that the infected patients present, in particular the elderly or those suffering from multiple comorbidities. Human pathogenic coronaviruses, some already identified since the 1960s and others more recently, are seven and they are divided into alpha and betacoronaviruses: 229E, OC43, NL63, HKU1, MERS-CoV, SARS-CoV, SARS-CoV-2. We describe the case of a male patient suffering from probable myocarditis due to subministration of monoclonal antibody Pembrolizumab and contextual isolation from nasopharyngeal swab of coronavirus NL63.

Keywords: Myocarditis; NL63 Coronavirus; COVID-19; Pembrolizumab

\section{Introduction}

Myocarditis is an inflammatory pathology of the heart muscle having heterogeneous etiology: infectious, autoimmune (e.g. Rheumatoid Arthritis, Sarcoidosis, Systemic Lupus Erythematosus, and Scleroderma) or iatrogenic for exposure to toxins or drugs (e.g. cardiotoxicity from chemotherapy [1]). Myocarditis presents itself with a wide spectrum of clinical manifestations, ranging from the infarct-like forms, heart failure, syncope up to hypo or hyperkinetic arrhythmias, or run in a paucisymptomatic way with almost silent symptoms. The most common cause of myocarditis is a viral infection. The most identified etiological agents are: Adenoviruses, Parvovirus B19, Coxsackie viruses or other Enteroviruses, Epstein-Barr virus, Cytomegalovirus, Rubella virus, HIV, HCV or polioviruses. Bacteria, fungi or parasites may also be involved. The coronavirus SARS-CoV2, the causative agent of COVID 19, which is responsible for the current pandemic, has a close correlation with myocardial damage and the increased risk of cardiovascular events that the infected patients present, in particular the elderly or those suffering from multiple comorbidities. Human pathogenic coronaviruses, some already identified since the 1960s and others more recently, are seven and they are divided into 


\section{Epidemiology International Journal}

alpha and betacoronaviruses: 229E, OC43, NL63, HKU1, MERS-CoV, SARS-CoV, and SARS-CoV-2. We describe the case of a male patient suffering from probable myocarditis due to sub ministration of monoclonal antibody Pembrolizumab and contextual isolation from nasopharyngeal swab of coronavirus NL63.

\section{Clinical Case}

Male patient, Italian, 79 years old, suffering from advanced prostate adenocarcinoma with bone and lymph node metastases and in therapy with Pembrolizumab, humanized anti-PD-1 monoclonal antibody. The patient came to our attention on the oncologist's advice, following the evidence of signs and symptoms of heart failure and the contextual electrocardiographic relief of bifascicular ventricular tachycardia (Figure 1).

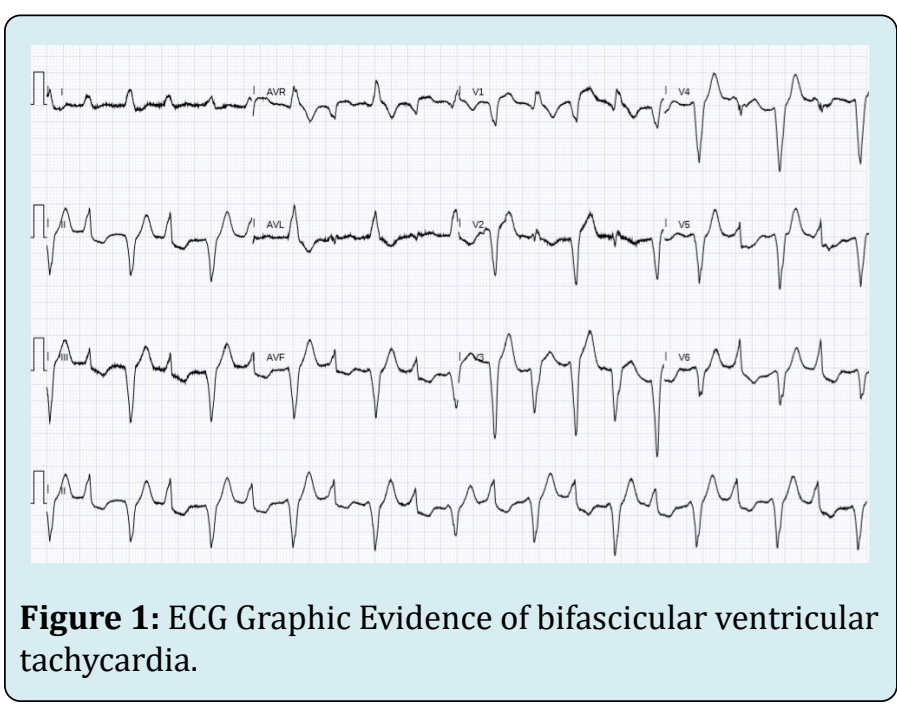

The echocardiogram showed a left ventricle of normal volume with severe reduction of the global contractile function for diffuse hypokinesia (FE 30\%), in the absence of a significant valve and pericardial effusion. The right sections appeared within the limits of the norm. Blood tests showed high values of high sensitivity troponin $(10024 \mathrm{ng} /$ $\mathrm{ml}$ ) and of myocardionecrosis enzymes as probable ischemic heart disease, neutrophilic leukocytosis with lymphopenia, ESR $38 \mathrm{~mm} / \mathrm{h}$ and PCR $11.93 \mathrm{mg} / \mathrm{dl}$. We performed a coronarographic examination, as an emergency measure, and the examination did not show significant coronary stenosis, therefore directing clinical suspicion towards myocarditis. The patient also reported cough and flu-like symptoms which were also present prior to hospitalization. He had come into contact with potentially COVID positive subjects due to his stay in areas with high endemia. The patient was then subjected to HRTC (Figure 2), which showed areas of parenchymal consolidation with lobar atelectasis, double nasopharyngeal swab and serological test for SARS-CoV2 research, which were all negative. Bacteriological tests on sputum sample and urinary antigens for Legionella and Pneumococcus were negative. The patient underwent plasma and serum sampling in case he showed signs of the genome or antibodies of the common viruses involved in the etiopathogenesis of myocarditis but all tests were negative. Excluding COVID or another viral etiology, the patient underwent high-dose corticosteroid therapy suspecting myocarditis during therapy with Pembrolizumab.

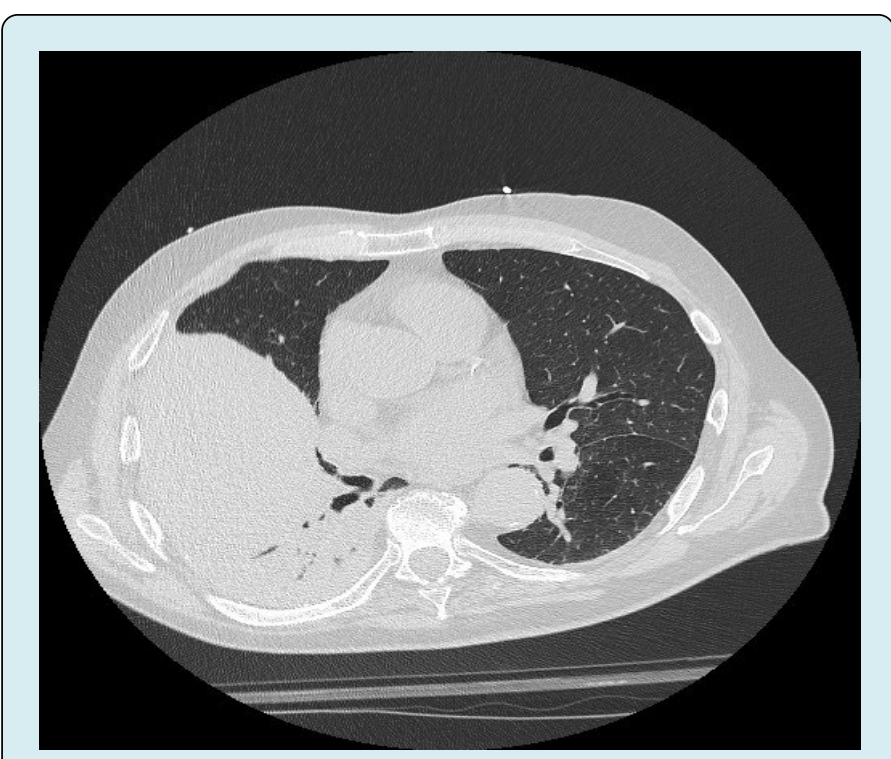

Figure 2: Evidence at HRTC parenchymal consolidation with morphological characteristics of the atelectasis type at the level of the lower right lobe.

During hospitalization, the patient had several episodes of sustained ventricular tachycardia, some of them undergoing DC-Shock. Subsequently, we were informed of a late positivity of the nasopharyngeal swab not for SARS-CoV2 but for coronavirus NL63 (Real-Time PCR Seegene Anyplex II RV 16 Detectio V1.1), in the absence of respiratory or gastroenteric symptoms related to a viral etiology. The patient presented a slow and gradual recovery of cardiac contractility despite serious clinical conditions and poor life expectancy. The predischarge echocardiographic control, after prolonged highdose steroid therapy, showed a left ventricle of normal size with preserved global and longitudinal systolic function. The heart rhythm was sinus, in the absence of ventricular ectopias (Figure 3). In agreement with our oncologist, and given an estimated life expectancy of less than 6 months, the patient did not undergo an endomyocardial biopsy despite its known diagnostic utility. At discharge, inflammation indices and myocardialnecrosis enzymes were normalized [2]. 


\section{Epidemiology International Journal}

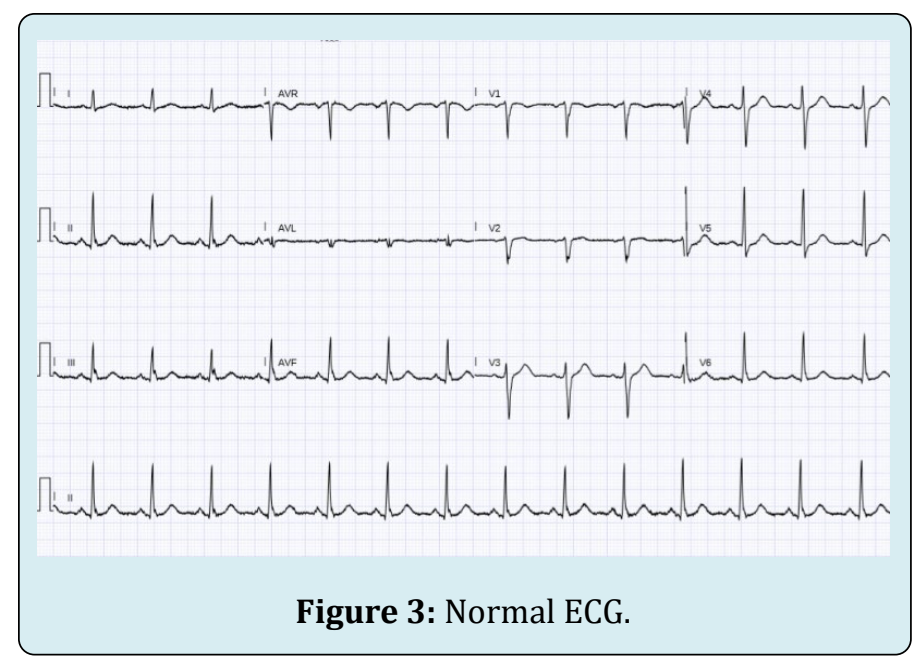

\section{Discussion}

In accordance with the criteria of the European Society of Cardiology, endomyocardial biopsy represents the "Gold Standard" examination for the diagnosis of myocarditis. Cardiac magnetic resonance imaging can be considered a support investigation. In our case, given the closure of the secondary cardiological diagnostic services during the COVID emergency, it was not possible to perform the resonance neither during hospitalization nor afterwards, since the patient died two weeks after discharge. Endomyocardial biopsy would certainly have been helpful in clarifying the diagnosis of myocarditis and its etiology. HCoV-NL63 is an ubiquitous virus, first isolated in 2004 in a 7-monthold baby with bronchiolitis, generally the cause of upper respiratory tract infections (coughing, but throat-related, rhinitis), in particular, during winter months and especially in children under 3 years of age. In the literature "croups" or laryngotracheobronchitis, bronchiolitis or severe interstitial pneumonia pictures with coronavirus isolation NL63 requiring oxygen support are rarely described. An earlier correlation between the virus and the Kawasaki disease had been reported subsequently decayed due to the lack of clinical data. Like other coronaviruses, NL63 has a direct tropism for the intestinal mucosa that results in the onset of symptoms such as nausea, vomiting and diarrhea, often described also during MERS-CoV Middle Eastern Respiratory Syndrome. The coronavirus NL63 is often the cause of coinfections with other respiratory viruses such as influenza, parainfluenza or metapneumovirus viruses. Studies show that NL63-induced immunodepression would pave a way for infections involving the upper or lower airways induced by other viruses. Our research indicates that currently there are no cases of myocarditis or pericarditis induced by coronavirus NL63 unlike the SARS-CoV2 virus which would seem to induce both direct damage to the heart tissue, through binding to the ACE2 receptor, and immuno-mediated by activation of the so-called "Cytokine Storm". HCoV-NL63 has also been frequently isolated from the respiratory secretions of asymptomatic subjects [3].

In terms of cardiotoxicity, only few cases on the association between pembrolizumab and myocarditis are available in the published literature; among them a case of cardiac arrest and another case of heart failure related to myocarditis are described $[2,4]$.

\section{Conclusion}

An emergency situation, such as the one that has swept us apart since January 2020, shows us that we often focus our attention on the most probable etiological causes, leaving out other aspects of medicine. In our case, in fact, if we had found ourselves outside the current COVID emergency, a report of Pembrolizumab myocarditis would have been sent sooner, steroid therapy would have been started and the patient would never have been declared a coronavirus test. Are we sure that coronavirus cases which are considered "minor" should not be treated as serious clinical pictures at the level of the respiratory or extra-respiratory tract, perhaps not only in childhood but also in the adult subject, in this case immunosuppressed for the treatment with Pembrolizumab? Isn't it worth regularly researching the aforementioned viruses and directing pharmacological research towards the creation of potential therapies or vaccines against viruses that are perhaps underestimated and possible causes of serious diseases?.

\section{References}

1. Mahmood SS, Fradley MG, Justine V Cohen, Nohria A, Reynolds KL, et al. (2018) Myocarditis in Patients Treated With Immune Checkpoint Inhibitors. J Am Coll Cardiol 71(16): 1755-1764.

2. Inayat F, Masab M, Gupta S, Ullah W (2018) New Drugs and New Toxicities: Pembrolizumab-Induced Myocarditis. BMJ Case Rep 2018.

3. Fielding BC (2011) Human coronavirus NL63: a clinically important virus?. Future Microbiol 6(2): 153-159.

4. Läubli H, Balmelli C, Bossard M, Pfister O, Glatz K, et al. (2015) Acute heart failure due to autoimmune myocarditis under Pembrolizumab treatment for metastatic melanoma. J Immunother Cancer 3: 11. 